\title{
AccuPower: An Accurate Power Estimation Tool for Superscalar M icroprocessors*
}

\author{
D mitry Ponomarev, Gurhan K ucuk and K anad Ghose \\ Department of Computer Science \\ State U niversity of N ew Y ork, Binghamton, NY 13902-6000 \\ e-mail:\{dima, gurhan, ghose\}@cs.binghamton.edu \\ http://www.cs.binghamton.edu/ low power
}

\begin{abstract}
This paper describes the AccuP ower toolset - a set of simulation tools accurately estimating the power dissipation within a superscalar microprocessor. AccuPower uses a true hardware level and cycle level microarchitectural simulator and energy dissipation coefficients gleaned from SPICE measurements of actual CM OS layouts of critical datapath components. Transition counts can be obtained at the level of bits within data and instruction streams, at the level of registers, or at the level of larger building blocks (such as caches, issue queue, reorder buffer, function units). This allows for an accurate estimation of switching activity at any desired level of resolution.
\end{abstract}

The toolsuite implements several variants of superscalar datapath designs in use today and permits the exploration of design choices at the microarchitecture level as well as the circuit level, including the use of voltage and frequency scaling. In particular, the AccuP ower toolsuite includes detailed implementations of currently used and proposed techniques for energy/power conservations including techniques for data encoding and compression, alternative circuit approaches, dynamic resource allocation and datapath reconfiguration. The microarchitectural simulation components of AccuP ower can be used for accurate evaluation of datapath designs in a manner well beyond the scope of the widely-used Simplescalar tools.

\section{Introduction}

It has been recognized by the processor design community that power dissipation is a first-class architectural design constraint not only for portable computers and mobile communication devices, but al so for high-performance superscalar microprocessors [10]. A fair amount of research efforts have been directed towards reduction of power dissipation in this high-end systems. Proposed solutions include both microarchitectural and circuit-level techniques.

\footnotetext{
* supported in part by DARPA through contract number FC 306020020525 under the PAC-C program, and the NSF through award no. MIP 9504767 \& EIA 9911099.
}

Several power estimation tools for processors have been designed, including Wattch [2], Simplepower [15] and TEM ${ }^{2} P^{2} E S T$ [4] to name a few. Simplepower is used only for simple 5-stage scalar pipelines and only models the execution of integer instructions; it is not applicable to superscalar processors. The major drawback of other tools is their reliance on the Simplescalar simulator [1], which lumps many critical datapath artifacts like the issue queue (IQ), the reorder buffer (ROB) and physical register files (PRFs) into a unified structure called R egister U pdate U nit (RUU), quite unlike the real implementations, where the number of entries and the number of ports in all these structures are quite disparate. Consequently, power dissipation can not be estimated accurately on a per-component basis. Considering that in many cases, these components collectively contribute to more than half of the overall power dissipation of the chip [5, 12], it is necessary to have facilities that allow the design space of these components and their interactions be modeled as accurately as possible. This is exactly one of the areas where A ccuPower provides a more accurate simulation and power estimation framework than existing public domain tools.

Needless to say, accurate power estimation tools are available within the organizations of individual microprocessor vendors for specific architectures, but they are not available to the public and/or their distribution is limited. Examples of such tools include IBM 's MET and its associated power estimation components for a Power PC implementation and Compaq's A SIM tool for simulating and estimating transition activity within Alpha processor implementations [3]. Tensilica's OEM-customizable $X$ tensa core comes with a power estimation tool that provides reasonably accurate power estimation for customized datapaths. Other power estimation tools, mainly targeting relatively simple embedded CPU s have also been developed, such as the one in [7].

Specifically, the main features of the AccuPower toolset are as follows:

- Accupower uses detailed cycle-level simulation of all major datapath components and interconnections that mimic the actual hardware implementation, including separate and realistic implementations of the issue queue, register files, ROB, load-store queue and forwarding mechanisms. 
- Detailed and accurate simulations of the on-chip cache hierarchy (including multiple levels of on-chip caches, interconnections, arbitration and chip-level I/O traffic) are an intrinsic part of A ccuPower.

- A significant part of a modern processor's power dissipation can occur in the I/O pads. Collectively, CPU-internal interconnections for explicit data transfers and forwarding and the clock distribution network are also significant sources of power dissipation within superscalar processors. A ccuPower models these interconnections in great detail, including the connections themselves as well as associated ports on datapath components and drivers.

- The A ccuPower tool supports built-in models for three major variants of superscalar datapaths in wide use.

- Accupower includes well-instrumented facilities for collecting datapath statistics of rel evance to both power and performance at the level of bits, bytes (for data and instruction flows) within logic blocks and subsystem-level components and the entire processor.

- Implementations of cutting-edge techniques for power/ energy reduction at the microarchitectural level, at the logic level and circuit level, as well as techniques based on clock gating, voltage and frequency scaling to facilitate the exploration of the design space are included in A ccuPower.

- AccuPower uses energy/power dissipation coefficients associated with the energy dissipating events within each key datapath component and the interconnections. These are combined with the transition counts obtained form the microarchitectural simulation component to get the overall energy/power dissipation figures. The coefficients could be estimated analytically from process-specific electrical parameters by adapting tools such as CACTI [15]. A more accurate approach, and one also supported in A ccupower is to derive these coefficients from SPICE measurements of actual layouts of these components. The Accupower toolkit includes representative VLSI layouts of some key datapath components and the dissipation coefficients estimated using SPICE for these components. Coefficients for leakage dissipations are also provided.

We believe that short of an actual implementation, AccuPower's power estimation strategy, based on SPICE-measured dissipation coefficients and detailed cycle-by-cycle, true hardware level simulation, is as accurate as it gets. A s part of an ongoing effort, we are al so in the process of validating the energy dissipation coefficients of critical datapath components from actual subscal eimplementations through M OSIS. A release of the AccuPower toolset, including the VLSI layouts and SPICE - measured data is planned for in the near future. The planned release will also incorporate models for multi-clustered datapaths; this feature is being implemented in AccuPower at this point.

\section{Variants of M odern Superscalar Datapaths}

Three variations of superscalar datapaths are predominantly in use in modern implementations. They mainly differ in two respects: how the physical registers are implemented and when the readout of the source physical registers occurs.

Datapath A (Figure 1): Here, input registers that contain valid data are read out while the instruction is moved into the IQ. As the register values required as an input by instructions waiting in the IQ (and in the dispatch stage) are produced, they are forwarded through forwarding buses that run across the length of the IQ [11]. The issue queue

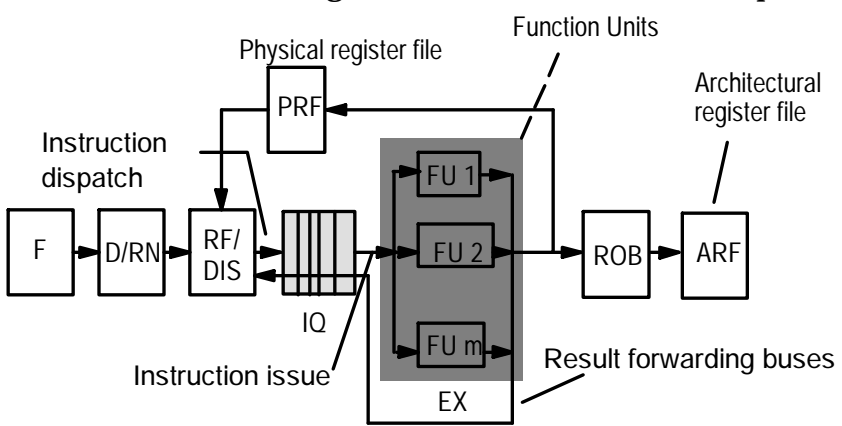

Figure 1. Datapath $A$

entry for an instruction has one data field for each input operand, as well as an associated tag field that holds the address of the register whose value is required to fill the data field. When a function unit completes, it puts out the result produced along with the address of the destination register for this result on a forwarding bus. Comparators associated with each IQ entry then match the tag values stored in the fields (for waited-on register values) against the destination register address floated on the forwarding bus [11]. On a tag match, the result floated on the bus is latched into the associated input field. Since multiple function units complete in a cycle, multiple forwarding buses are used; each input operand field within a IQ entry thus uses a comparator for each forwarding bus. Examples of processors using this datapath style are the IBM Power PC 604, 620 and the HAL SPARC 64 [9].

Datapath B (Figure 2): Here, even if input registers for an instruction contain valid data, these registers are not read out at the time of dispatch. Instead, when all the input operands of an instruction waiting in the IQ are valid and a function unit of the required type is available, all of the input operands are read out from the register file (or as they are yet to be written to the register file, using bypassing logic to forward data from latter pipeline stages) and the instruction is issued. In this case, the IQ entry for an instruction is considerably narrower compared to the IQ entries for Datapath A, since entries do not have to hold input register values. The dispatch/issue logic can be implemented using a global scoreboard that keeps track of instructions and register/FU availability. A Iternatively, an associative logic similar to that of Datapath $A$ can be used to update the status of input registers for instructions waiting within the IQ (as shown in Figure 2). Examples of 
processors using this datapath style are the MIPS 10000 , MIPS 12000 and the DEC 21264 [9].

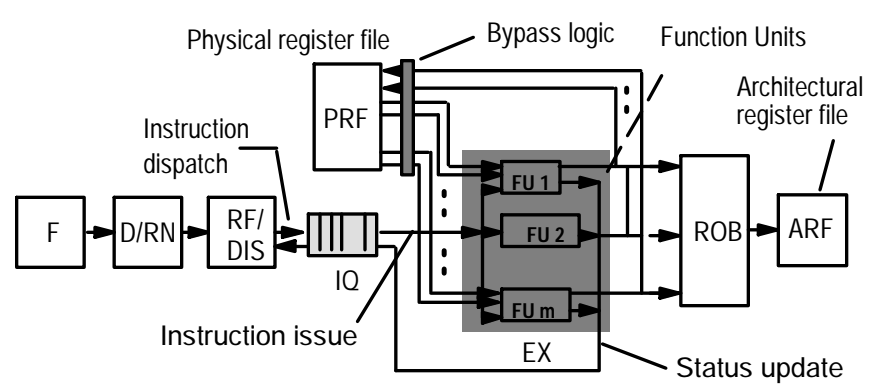

Figure 2. Datapath B

Datapath C (Figure 3): Here, the ROB entry set up for an instruction at the time of dispatch contains a field to hold the result produced by the instruction - this serves as the analog of a physical register. We assume that each $\mathrm{ROB}$ entry may hold only 32 -bit long result, thus requiring the allocation of two ROB entries for an instruction producing a double-precision value. A dispatched instruction attempts to read operand values either from the A rchitectural Register File (ARF) directly (if the operand value was committed) or associatively from the ROB (from the most recently established entry for an architectural register, in case the operand value was generated but not committed. Source registers that contain valid data are read out into the IQ entry for the instruction. If a source operand is not available at the time of dispatch in the ARF or the ROB, the address of the physical register (i.e., ROB slot) is saved in the tag field associated with the source register in the IQ entry for the instruction. Forwarding to the waiting IQ slots is performed similar to Datapath A. Examples of processors using this datapath style are the Intel Pentium II and Pentium III. [9]. Two variants of Datapath $C$ are implemented: one where a rename table is used to look up source operands from the ROB and another where associative searching is used to locate the most recently generated value into an architectural register from the ROB.

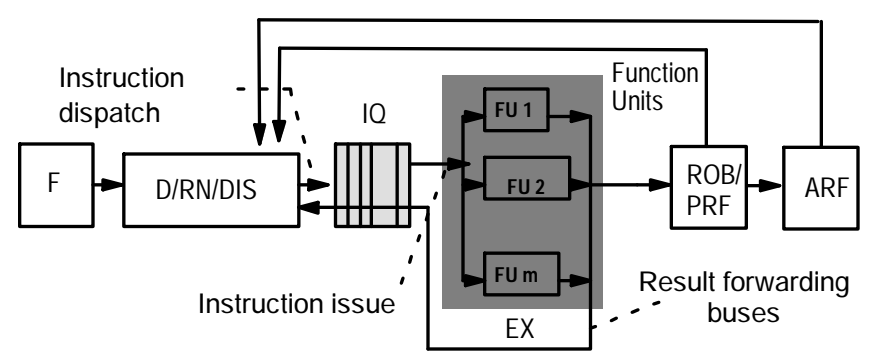

Figure 3. Datapath C

\section{Implementation}

The A ccuPower tool consists of the three components: a microarchitectural simulator, which is a greatly modified version of the Simplescalar; the VLSI layouts for major datapath components and caches in a 0.5 micron process; and power estimation module that uses coefficients obtained from the SPICE simulations in conjunction with transition counts obtained from the microarchitectural simulator to compute energy/power. (The public release planned will use 0.18 and 0.25 micron layouts as well.) Figure 4 summarizes the overall power/energy estimation methodology used in A ccuPower.

\subsection{Microarchitectural Simulators}

To support the three superscalar datapath configurations discussed in section 2, we significantly modified the Simplescalar simulator [1] and implemented separate structures for the IQ, the ROB, the rename table, the physical register files and the architectural register files. Three versions of the simulator were designed - one for each datapath configuration discussed above. We also accurately modeled the pipeline structures and various interconnections within the datapath, namely the dispatch buses, issue buses, result buses and commit buses. In a typical superscalar processor, multiple sets of such buses are needed to sustain the dispatch/issue/commit rate. Traffic on each such bus and read/write activity within the register files implementing the datapath storage components are separately monitored and analyzed as discussed later.

The extent of our modifications to the Simplescalar can be gauged by the fact that barely $10 \%$ of the original code was retained. The sim-outorder.c file was completely rewritten (with the exception of the fetch stage) to support a true cycle-by-cycle out-of-order instruction execution. This is in contrast to the original Simplescalar code, where instructions are actually executed in-order at the dispatch stage and the effects of out-of-order execution are implemented through the convoluted manipulations with the RUU. Significant modifications have also been incorporated into the cache simulator, as discussed below. Specifically, the changes that were made to the Simplescalar to simulate the realistic superscal ar pipelines are as follows:

(i) We split the monolithic cache access stage as used in Simplescalar into two stages to mimic the real-world situation where cache accesses - even L 1 cache accesses are typically performed in multiple cycles and provided a support for pipelined cache.

(ii) We modeled the contention for the bus between $L 1$ and $L 2$ caches. This is important because the $L 1$ caches are typically split with separate caches for instructions and data. L2 cache, however, is typically unified. Situation when both I1 miss and D1 miss occur in the same cycle are certainly possible and they require proper modelling to arbitrate for the access to the L 2 cache. 
(iii) A long similar lines, we modelled the contention for the off-chip interconnection from the L 2 cache to the DRAM modules.

(iv) The decode stage of the Simplescalar datapath, where instruction dispatching and register renaming is performed, was split into two stages, as it is unrealistic to perform the fairly complicated operations of dispatching, register renaming and source register readout in a single cycle.

(v) We also assumed realistic delays on the interconnections, noting that it takes a full cycle to distribute the result produced by one of the FUs to the waiting operands in the issue queue (D atapaths $A$ and $C$ ).

To summarize, we attempted to design a simulator that would closely mimic the actual microarchitecture and hardware implementations of real CPUs on a cycle-by-cycle basis. The focus of the A ccuPower tool is to facilitate the exploration of the design space of superscalar processors and gauge the impact of well-used and cutting-edge techniques for saving power and/or energy. The tool also supports the exploration of circuit-level techniques and the more standard power reduction techniques like voltage and frequency scaling.

\subsection{Choosing the Estimation Accuracy}

AccuPower offers the user several flexibilities in controlling the accuracy of the power estimation and the simulation time. The accuracy of the power estimations is a function of the detail with which transition counts are obtained and also a function of the accuracy of the dissipation coefficients used in the computation of the energy/power. Where the accuracy requirements are not exacting, facilities are provided for turning down the extent of transition counting. As examples, one can turn off detailed transition counting at the level of bits, occupancy counting within resources such as the IQ, ROB, and transition counting within datapath components not of interest.

In addition to having a control over the extent of transition counting as described above, it is useful to have a choice on the accuracy of the dissipation coefficients used. For the best accuracy, coefficients obtained from SPICE measurements of actual VLSI layouts can be used. This is clearly not a viable approach for exploring the entire design space, as layouts have to be altered with each technology generation and with each variation in the implementation. The A ccuPower infrastructure therefore offers an alternative in the form of parameterized models of energy dissipation for major datapath components. The parameters used include the word size, number of words/registers, number of ports etc. The parameterized models are used to get an approximate idea of the dissipation coefficients that have to be used when different-sized components are required. The base case values to use in these parameterized model s can come from SPICE measurements of actual layouts for the base case or by analytically estimating the capacitive coefficients using technology parameters based on a good idea of the layout.

\subsection{VL SI Layouts: G etting Accurate C oefficients}

For accurately estimating the energy/power for the key datapath components and power of a processor as a whole using AccuPower, the transition counts and event sequences gleaned from the microarchitectural simulator are used, along with the energy dissipations for each type of event, as measured from the actual VLSI layouts using SPICE. CMOS layouts for the on-chip caches, IQ, PRF, $A R F$ and $R O B$ in a 0.5 micron 4 metal layer CM OS process (HPCMOS-14TB) were used for the key datapath components to get an accurate idea of the energy dissipations for each type of transition. We are in the process of migrating these layouts to 0.18 micron process. The results for the 0.5 micron layouts are, however, quite representative, al though greatly scaled down compared to what one would see with 0.18 micron implementations running at a faster clock rate! The exception to this claim are the wire dissipations outside these components. (Dissipations on such wires at small feature sizes become relatively dominant.)

The register files that implement the $R O B$ and I Q were carefully laid out in the 0.5 micron process to optimize the dimensions and allow the use of a $300 \mathrm{M} \mathrm{Hz}$ clock. A V dd of 3.3 volts is assumed for all the measurements. (The value of the clock rate was constrained by the cache cycle time, as determined by the cache layouts for a two-stage pipelined cache in the same technology.) In particular, these register files feature differential sensing, limited bit line driving and pulsed word line driving to save power. A ugmentations to the register file structures for the IQ (mainly in the form of comparators with each of the 3 source operand fields and the four result/tag buses) are also fully implemented; a pulldown comparator is assumed for associative data forwarding to entries within the IQ and the device sizes were carefully optimized to strike a balance between the response speed and the energy dissipations. For each energy dissipating event, SPICE measurements were used to determine the energy dissipated. These measurements are used in conjunction with the transitions counted by the hardware-level, cycle-by-cycle simulator to estimate dynamic energy/power accurately. A ctual layout data was al so used for estimating the leakage power of the layouts in the smaller feature sizes.

\subsection{Speeding up the Execution - M ultithreading}

The instrumentation needed to determine the bit level activities within data flow paths and data storages (both explicit and implicit) and log all major switching activities slows down the simulation drastically. To get a reasonable overall simulation performance with all the instrumentation in place, we resorted to the use of multithreading. Specifically, we use a separate thread for the data stream analysis, as shown in Figure 4. The two-threaded implementation is run on SMPs to get an acceptable simulation speed. The data acquired from basic 
instrumentation within the main simulation thread is buffered and fed into a separate thread where it was further analyzed.

With a single thread implementing all of the simulation, instrumentation and analysis, the overall simulation speed was reduced by as much as $40 \%$ compared to the original Simplescalar simulation without any modification and instrumentation. With both threads in place as shown in Figure 4, and with the use of inter-thread buffers of an optimized size, the overall simulation time achieved was often significantly better on a SM P compared to the single-threaded implementation. The performance of the dual-threaded version was also acceptably close to that of the original Simplescalar simulator without any of the enhancements and the instrumentation.

A public release of A ccuPower is planned in the very near future. Validations of the SPICE - measured data from MOSIS-supported implementations of some critical datapath components are al so planned as part of an ongoing effort.

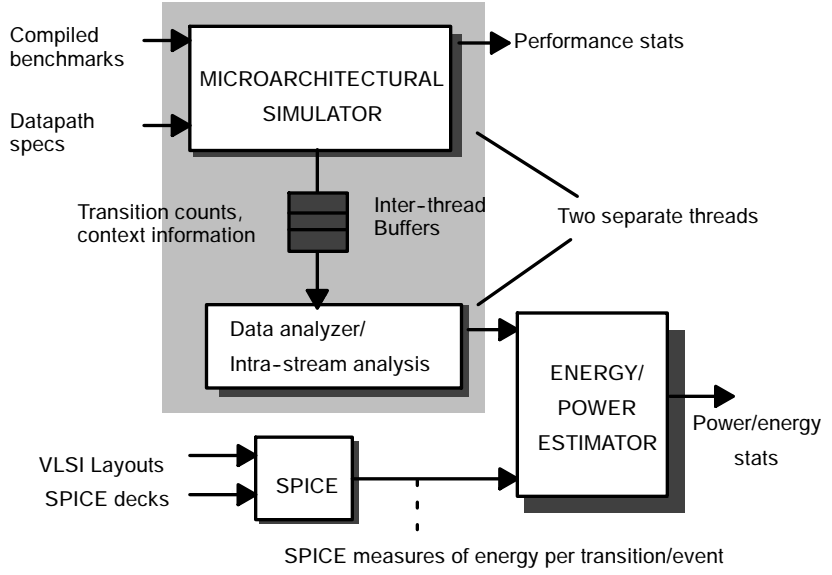

Figure 4. Power Estimation Methodology

\section{Using AccuPower}

AccuPower can be used to obtain realistic measurements pertaining to power and performance in a variety of ways. These include:

1) Raw data collection. This falls into two categories:

a) The tool monitors the bit-level datapath activity on the interconnections, dedicated transfer links, and read/write activity of the register files that implement the datapath storage components. We also provide the data analyzer (implemented as a separate thread for performance reasons, as discussed above) to examine the collected data streams on the presence of zero-bytes. Presence of zero bytes has been exploited to reduce the switching activity and hence power dissipations in caches [11] and in other datapath components [6]. In addition, the data anal yzer estimates the percentage of bits that did not change their values since the previous value had been driven on the same link. Considerable power savings can be achieved on the datapath interconnections by not driving such bits. [6]. To further reduce the number of bits driven on the interconnections, such bit-slice invariance can be used on top of zero-byte encoding. Representative resulting percentages of bits that do not have to be driven on the datapath interconnections are shown for Datapaths A and $B$ in Figures 5 and 6 respectively.

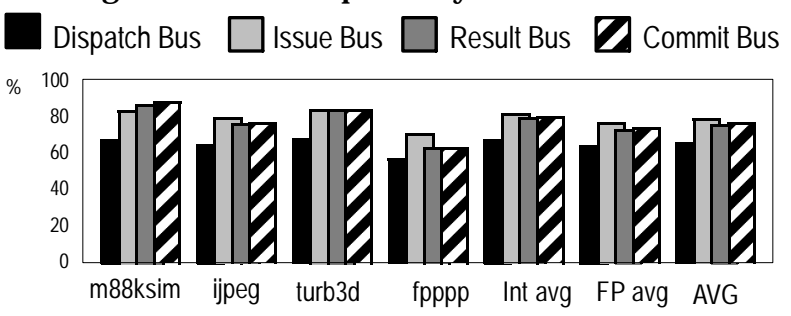

Figure 5. $\%$ of bits not driven using bit-slice invariance on top of zero byte encoding for Datapath A

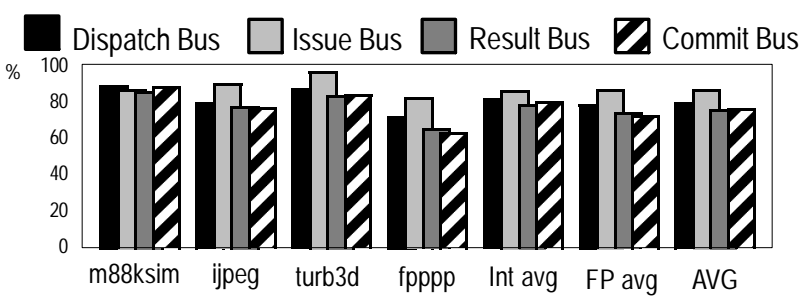

Figure 6. $\%$ of bits not driven using bit-slice invariance on top of zero byte encoding for Datapath $B$

b) In AccuPower, the occupancies of individual datapath resources, as measured by the number of valid entries, are monitored and recorded. This capability is currently implemented for the IQ, the ROB, and the LSQ. Support for caches will be added in the near future. Figure 7 shows the occupancies of the IQ, the ROB, and the LSQ obtained from the execution of fpppp benchmark on A ccuPower simulator. Resource occupancies are recorded at every cycle and the averages have been taken for every 1 million of simulated cycles. This occupancy statistics can be used to drive the decision to dynamically allocate datapath resources depending on the application's needs. For example, if sampling of the resource occupancy indicates that the resource is currently overcommitted, some parts of this resource can be temporarily turned-off thus saving power dissipated within this resource. Such dynamic resizing of multiple datapath resources with minimal impact on performance has been studied in [12]. Representative results are shown in Figure 8. A ccuPower is the only currently available power estimation tool that allows to measure the occupancies and the datapath 
resource usages in general independently and thus explore microarchitectural techniques that exploit occupancy variations for power savings.

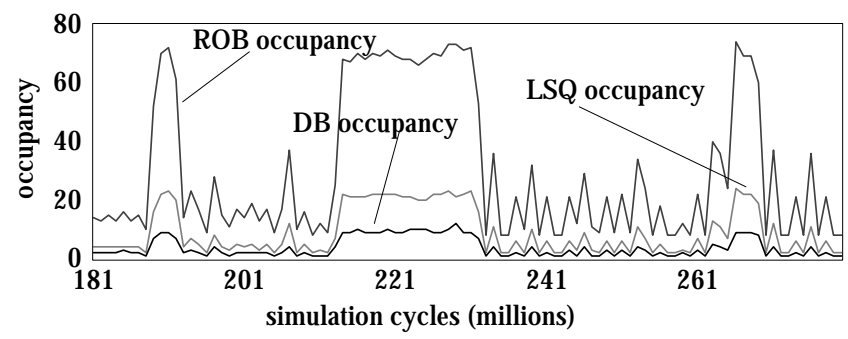

Figure 7. The occupancies of the ROB, the $D B \&$ the LSQ

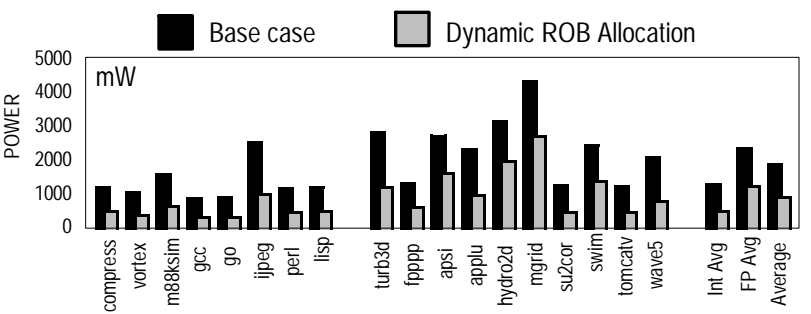

Figure 8. Total power dissipation within the $R O B$ for a 4-way superscalar processor. (Datapath C)

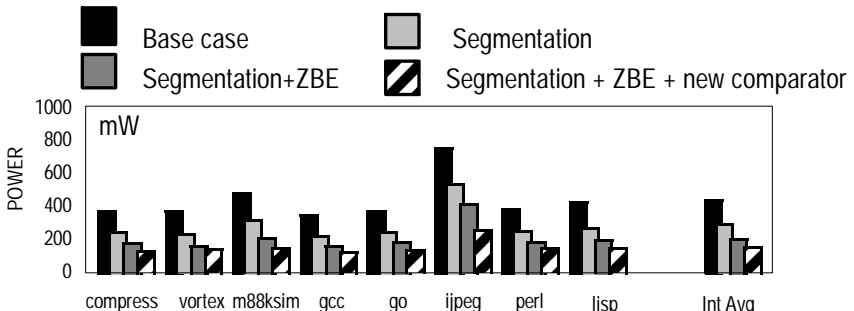

Figure 9. Total power dissipation within DB using bitline segmentation, power efficient comparators and zero-byte encoding (ZBE). (Only SPEC 95int results for datapath A are shown)

2) Accurate datapath component-level power estimation. Here, we use transition counts obtained from the architectural simulation in conjunction with the capacitance coefficients obtained from SPICE simulations. Figures 8 and 9 represent some typical measurements.

3) Exploration of various power reduction techniques. These include the use of zero-byte encoding (ZBE) [13], dynamic resource allocation using occupancy sampling [12] and the use of partitioned datapath components [16]. Figures 5,6 and 8 show some representative measurements.

4) Exploration of alternative circuit-level techniques. These currently include the use of fast dissipate- on- match comparators for associative lookup within the IQ, the ROB and the TL $B$, bit-linesegmentation within register files and zero-byte encoding [8]. Figure 9 demonstrates the representative savings, as measured by A ccuPower.

5) Explorations of alternative datapath architectures. As mentioned above, three datapath configurations are currently supported. Figures 5 and 6 can be used to compare the bit-slice inactivities in Datapaths A and B.

\section{Conclusions}

This paper presented AccuPower - an accurate simulation tool for estimating power dissipation within various flavors of modern superscalar datapaths on a per-component basis. AccuPower consists of microarchitectural simulator in the form of significantly redesigned version of the Simplescal ar simulator, the actual VLSI layouts for the key components of a superscalar processor, parameterized dissipation models and a power/energy estimator module. A ccuPower can be used to estimate power savings achieved by several proposed microarchitectural and circuit-level techniques and also to study the impact of microarchitectural innovations in general. A variety of superscalar datapath models are built into A ccuPower to facilitate such studies.

\section{R eferences:}

[1] Burger, D., and A ustin, T. M., "The Simplescalar tool set: Version 2.0", Tech. Report, Dept. of CS, U niv. of Wisconsin-M adison, June 1997 and documentation for all Simplescalar releases (through version 3.0).

[2] B rooks, D., Tiwari, V., M artonosi, M., "Wattch: A Framework for A rchitectural-L evel Power A nalysis and Optimizations", 27th International Symposium on Computer Architecture, 2000.

[3] Compaq Computer Corporation, The ASIM M anual, 2000.

[4] D hodapkar, A ., L im, C., Cai, G., D aasch R, "TEM 2P2E ST: A Thermal E nabled M ulti-M odel Power/Performance EST imator", in PA CS Workshop, held in conjunction with A SPL OS, 2000.

[5] Folegnani, D., Gonzal ez, A., "Energy-Effective Issue L ogic", 28th Int'l. Symposium on C omputer Architecture, July, 2001.

[6] Ghose, K., Ponomarev, D., K ucuk, G., Flinders, A ., K ogge, P., and Toomarian N.,"Exploiting Bit-slice Inactivities for Reducing Energy Requirements of Superscalar Processors," in Proc. of K ool Chips Workshop, M icro-33, 2000.

[7] Hsieh, C-T., Chen, L-S, and Pedram, M., "M icroprocessor Power A nalysis by Labeled Simulation", in Proc. DATE '01.

[8] Kucuk, G., Ghose, K., Ponomarev, D., Kogge, P., "Energy-Efficient Instruction Dispatch Buffer Design for Superscalar Processors", ISL PED-01, pp. 237-242.

[9] M icroprocessor R eport, various issues, 1996-2001.

[10] Mudge, T., "Power: A First-Class A rchitectural Design Constraint", IEEE Computer, A pril 2001, pp. 52-58.

[11] Palacharla, S., J ouppi, N. P. and Smith, J.E., "Quantifying the complexity of superscalar processors", Technical report CS-TR-96-1308, Dept. of CS, Univ. of Wisconsin, 1996.

[12] Ponomarev, D., Kucuk, G., Ghose, K., "Reducing Power Requirements of Instruction Scheduling Through Dynamic Allocation of Multiple Datapath Resources", 34th International Symposium on M icroarchitecture, D ecember, 2001, pp. 90-101.

[13] Villa, L., Zhang, M. and A sanovic, K., "Dynamic Zero Compression for Cache Energy Reduction", 33rd International Symposium on M icroarchitecture, December, 2000.

[14] Vijaykrishnan, N., Kandemir, M., Irwin, M.J. et al, "Energy-D riven Integrated $\mathrm{H}$ ardware-Software Optimizations Using SimplePower", 27-th International. Symposium on Computer A rchitecture, pp.95-106.

[15 ] Wilton, S., and J ouppi, N., "An Enhanced A ccess and Cycle Time M odel for On-Chip Caches", WRL Research Report 93-5, DEC WRL, 1994.

[16] Zyuban, V. and K ogge, P., "O ptimization of High-Performance Superscalar A rchitectures for Energy Efficiency", in Proc. of ISL PED, 2000, pp. 84-89. 